\title{
Global analysis of a delayed Monod type chemostat model with impulsive input on two substrates
}

\author{
Jianzhi Cao ${ }^{1 *}$, Junyan Bao ${ }^{1}$ and Peiguang Wang ${ }^{2}$
}

"Correspondence:

cjz2004987@163.com

'College of Mathematics and

Information Science, Hebei

University, Baoding, 071002,

P.R. China

Full list of author information is

available at the end of the article

\begin{abstract}
In this paper, a new Monod type chemostat model with delay and impulsive input on two substrates is considered. By using the global attractivity of a $k$ times periodically pulsed input chemostat model, we obtain the bound of the system. By the means of a fixed point in a Poincaré map for the discrete dynamical system, we obtain a semi-trivial periodic solution; further, we establish the sufficient conditions for the global attractivity of the semi-trivial periodic solution. Using the theory on delay functional and impulsive differential equations, we obtain a sufficient condition with time delay for the permanence of the system.
\end{abstract}

MSC: $34 \mathrm{~K} 45 ; 34 \mathrm{~K} 20$

Keywords: Monod type; globally attractivity; nutrient recycling; chemostat model

\section{Introduction and the model}

The chemostat is an important laboratory apparatus to study the growth of microorganism in a continuous environment. It has begun to occupy an increasing central role in ecological studies. As a tool in biotechnology, the chemostat plays an important role in bio-processing and the chemostat has many applications in waste water treatment, production by genetically altered organisms, etc. Chemostats with periodic inputs are studied in [1-7], those with periodic washout rate in [8, 9], and those with periodic input and washout in [10]. The structure assigned to the organisms in the model accounts for the dependence of the growth on the past history of the cells, and hence it is capable of predicting the lag phases and transient oscillations observed in experiments. Many authors have directly incorporated time delays in the modeling equations and, as a result, the models take the form of delay differential equations [11-22].

Many scholars pointed out that it was necessary and important to consider models with periodic perturbations, since these models might be quite naturally exposed in many real world phenomena (for instance, food supply, mating habits, harvesting). In fact, almost perturbations occur in a more-or-less periodic fashion. However, there are some other perturbations such as fires, floods, and drainage of sewage which are not suitable to be considered continuous. These perturbations bring sudden changes to the system. A chemostat model with time delays was first studied by Caperon [23] based on some experimental data. Unfortunately, the model proposed by Caperon created the possibility of a negative

(c) 2015 Cao et al. This article is distributed under the terms of the Creative Commons Attribution 4.0 International License (http://creativecommons.org/licenses/by/4.0/), which permits unrestricted use, distribution, and reproduction in any medium, provided you give appropriate credit to the original author(s) and the source, provide a link to the Creative Commons license, and indicate if changes were made. 
concentration of the substrate (nutrient). To correct this, Bush and Cook [24] investigated a model of the growth of one microorganism in the chemostat with a delay in the intrinsic growth rate of the organism but with no delay in the nutrient equation. They have also established that oscillations are possible in their model. Systems with sudden perturbations are involved in the impulsive differential equation, which have been studied intensively and systematically in $[25,26]$.

In this paper, we want to introduce and study a delayed Monod model system in a chemostat with periodically pulsed substrate and nutrient recycling on two substrates. The model takes the form

$$
\left\{\begin{aligned}
& \dot{S}_{1}(t)=-D S_{1}(t)-\frac{\mu_{1} S_{1}(t) x(t)}{\delta_{1}\left(k_{1}+S_{1}(t)\right)}, \quad t \neq n T, n \in Z_{+} \\
& \dot{S}_{2}(t)=-D S_{2}(t)-\frac{\mu_{2} S_{2}(t)(t)}{\delta_{2}\left(k_{2}+S_{2}(t)\right)}, \quad t \neq n T, n \in Z_{+} \\
& \dot{x}(t)=-D x(t)+e^{-D \tau_{1} \frac{\mu_{1} S_{1}\left(t-\tau_{1}\right) x\left(t-\tau_{1}\right)}{k_{1}+S_{1}\left(t-\tau_{1}\right)}} \\
& \quad+e^{-D \tau_{2}} \frac{\mu_{2} S_{2}\left(t-\tau_{2}\right) x\left(t-\tau_{2}\right)}{k_{2}+S_{2}\left(t-\tau_{2}\right)}, \quad t \neq n T, n \in Z_{+} \\
& S_{1}\left(t^{+}\right)=S_{1}(t)+p_{1}, \quad t=n T, n \in Z_{+} \\
& S_{2}\left(t^{+}\right)=S_{2}(t)+p_{2}, \quad t=n T, n \in Z_{+} \\
& x\left(t^{+}\right)=x(t), \quad t=n T, n \in Z_{+}
\end{aligned}\right.
$$

where $S_{1}(t), S_{2}(t)$ represent the concentrations of limiting substrates at time $t, x(t)$ denotes the plankton concentration at time $t, D$ is the washout rate; $p_{i}(i=1,2)$ denotes the input concentration of the limiting of pulsing; $\mu_{1}$ and $\mu_{2}$ are the uptake constants of the plankton; $\delta_{1}$ is the yield of the plankton per unit mass of the first substrate; $\delta_{2}$ is the yield of the plankton per unit mass of the second substrate. The constant $\tau_{i} \geq 0(i=1,2)$ denotes the time delay involved in the biomass depending on the conversion of nutrient to viable biomass. The positive constant, $e^{-D \tau_{i}}(i=1,2)$, is required, because it is assumed that the current change in biomass depends on the amount of nutrient consumed in $\tau_{i}(i=1,2)$ units of time in the past by the microorganisms that were in the growth vessel at that time and managed to remain in the growth vessel for the $\tau_{1}(i=1,2)$ units of time required to process the nutrient.

\section{Preliminaries}

In this section, we will give some notations and lemmas which will be used for our main results.

Let $R_{+}=[0, \infty), R_{+}^{3}=\left\{\left(x_{1}, x_{2}, x_{3}\right) \in R^{3}: x_{1}>0, x_{2}>0, x_{3}>0\right\} . S_{1}\left(n T^{+}\right)=\lim _{t \rightarrow n T^{+}} S_{1}(t)$, $S_{2}\left(n T^{+}\right)=\lim _{t \rightarrow n T^{+}} S_{2}(t), x\left(n T^{+}\right)=\lim _{t \rightarrow n T^{+}} x(t), S_{1}(t), S_{2}(t)$ are left-continuous at $t=n T$, $x(t)$ is continuous at $t=n T$.

Lemma 2.1 ([25, 26]) Consider the following impulse differential inequalities:

$$
\begin{aligned}
& \omega^{\prime}(t) \leq(\geq) p(t) \omega(t)+q(t), \quad t \neq t_{k}, \\
& \omega\left(t_{k}^{+}\right) \leq(\geq) d_{k} \omega\left(t_{k}\right)+b_{k}, \quad t=t_{k}, k \in N,
\end{aligned}
$$

where $p(t), q(t) \in C\left(R_{+}, R\right), d_{k} \geq 0$, and $b_{k}$ are constants.

Assume:

$\left(\mathrm{A}_{0}\right)$ the sequence $t_{k}$ satisfies $0 \leq t_{0}<t_{1}<t_{2}<\cdots$, with $\lim _{t \rightarrow \infty} t_{k}=\infty$;

$\left(\mathrm{A}_{1}\right) \omega \in P C^{\prime}\left(R_{+}, R\right)$ and $\omega(t)$ is left-continuous at $t_{k}, k \in N$. 
Then

$$
\begin{aligned}
\omega \leq(\geq) & \omega\left(t_{0}\right) \Pi_{t_{0}<t_{k}<t} d_{k} \exp \left(\int_{t_{0}}^{t} p(s) d s\right) \\
& +\sum_{t_{0}<t_{k}<t}\left(\Pi_{t_{k}<t_{j}<t} d_{j} \exp \left(\int_{t_{k}}^{t} p(s) d s\right)\right) b_{k} \\
& +\int_{t_{0}}^{t} \Pi_{s<t_{k}<t} d_{k} \exp \left(\int_{s}^{t} p(\theta) d \theta\right) q(s) d s, \quad t \geq t_{0} .
\end{aligned}
$$

Consider the following system:

$$
\left\{\begin{array}{l}
\dot{S}(t)=-D S(t), \quad t \neq n T(i=1,2), n \in Z_{+}, \\
S\left(t^{+}\right)=S(t)+p_{i}, \quad t=n T(i=1,2), n \in Z_{+} .
\end{array}\right.
$$

Lemma 2.2 System (2.2) has a positive periodic solution $u_{i}^{*}(t)=\frac{p_{i} \exp (-D(t-n T))}{1-\exp (-D T)}$ for all $t \in$ $(n T,(n+1) T], n \in Z_{+}$, which is globally uniformly attractive.

The proofs of Lemmas 2.1 and 2.2 are simple, we omit them here.

Lemma 2.3 ([27]) Consider the following delay differential equation:

$$
\frac{x(t)}{d t}=r_{1} x\left(t-\tau_{1}\right)+r_{2} x\left(t-\tau_{2}\right)-r_{3} x(t)
$$

where $r_{1}, r_{2}, r_{3}, \tau_{1}, \tau_{2}$ are all positive constants and $x(t)>0$ for $t \in[-\tau, 0]$.

(i) If $r_{1}+r_{2}<r_{3}$, then $\lim _{t \rightarrow \infty} x(t)=0$.

(ii) If $r_{1}+r_{2}>r_{3}$, then $\lim _{t \rightarrow \infty} x(t)=+\infty$.

Consider the following system:

$$
\left\{\begin{array}{l}
\dot{S}(t)=-S(t), \quad t \neq n T+\tau_{i}(i=1,2, \ldots, k), n \in Z_{+}, \\
S\left(t^{+}\right)=S(t)+p_{i}, \quad t=n T+\tau_{i}(i=1,2, \ldots, k), n \in Z_{+},
\end{array}\right.
$$

where $T$ is the period of the impulsive effect and $\tau_{0}=0<\tau_{1}<\tau_{2}<\cdots<k=T$ are for the $k$ times of impulsive effects per period $T$.

Lemma 2.4 The subsystem (2.3) has a positive periodic solution $\tilde{S}(t)$ and for every solution $S(t)$ of $(2.3)$ we have $|S(t)-\tilde{S}(t)| \rightarrow 0$ as $t \rightarrow \infty$, where

$$
\begin{cases}\tilde{S}(t)=S_{i}^{+} \exp \left(-\left(t-n T-\tau_{i-1}\right)\right), & t \in\left(n T+\tau_{i-1}, n T+\tau_{i}\right], \\ \tilde{S}(0)=S_{0}^{+}=\frac{\sum_{j=1}^{k} p_{j} \exp \left(\left(-T+\tau_{j}\right)\right)}{1-\exp (-T)}, & S_{i}=S_{i-1}^{+} \exp \left(-p_{i}\right), \quad i=1,2, \ldots, k .\end{cases}
$$

Lemma 2.5 Let $\left(S_{1}(t), S_{2}(t), x(t)\right)$ be any solution of system (1.1) with initial values $\left(S_{1}\left(0^{+}\right), S_{2}\left(0^{+}\right), x(0)\right) \in R_{+}^{3}$. There exists a constant $L>0$ such that $S_{1}(t)<L, S_{2}(t)<L$, $x(t)<L$.

Proof Let $\left(S_{1}(t), S_{2}(t), x(t)\right)$ be any solution of system (1.1) with initial value $\left(S_{1}\left(0^{+}\right), S_{2}\left(0^{+}\right)\right.$, $x(0)) \in R_{+}^{3}$. Define a function

$$
V(t)=\delta_{1} e^{-D \tau_{1}} S_{1}\left(t-\tau_{1}\right)+\delta_{2} e^{-D \tau_{2}} S_{2}\left(t-\tau_{2}\right)+x(t) .
$$


The upper right derivative of $V(t)$ along the trajectories of (1.1) is

$$
\left\{\begin{array}{l}
\dot{V}(t)=-D V(t), \quad t \neq n T+\tau_{i}(i=1,2), n \in Z_{+}, \\
V\left(t^{+}\right)=V(t)+\delta_{i} e^{-D \tau_{i}} p_{i}, \quad t=n T+\tau_{i}(i=1,2), n \in Z_{+} .
\end{array}\right.
$$

Without loss of generality, we assume that $\tau_{1}>\tau_{2}$. From the system (1.1), it is easy to see there exists a $M_{1}$ such that

$$
S_{1}(t)<M_{1}, \quad S_{2}(t)<M_{1}, \quad x(t)<M_{1},
$$

as $t \in\left[0, \tau_{1}\right] . V(t)$ is for 2 times of the impulsive effects per period $T$ as $t \in\left(\tau_{1}, \infty\right)$. Because $\tau_{1}>\tau_{2}$, there exists a $n_{0}$ such that

$$
\tau_{2}^{*} \doteq n_{0} T+\tau_{2} \in\left(\tau_{1}, \tau_{1}+T\right]
$$

Let

$$
\begin{aligned}
& \bar{\tau}_{1}=\tau_{2}^{*}-\tau_{1}, \quad \bar{\tau}_{2}=T-\bar{\tau}_{1}, \\
& \bar{p}_{1}=\frac{\delta_{2} e^{-D \tau_{2}} e^{-D \bar{\tau}_{2}} p_{2}+\delta_{1} e^{-D \tau_{1}} p_{1}}{1-e^{-D T}}, \quad \bar{p}_{2}=\frac{\delta_{1} e^{-D \tau_{1}} e^{-D \bar{\tau}_{1}} p_{1}+\delta_{2} e^{-D \tau_{2}} p_{2}}{1-e^{-D T}} .
\end{aligned}
$$

By Lemma 2.4, we obtain

$$
\left\{\begin{array}{l}
V(t)=\bar{p}_{1} e^{-D\left(t-n T-\tau_{1}\right)}, \quad\left(n T+\tau_{1}, \tau_{1}+n T+\tau_{1}^{*}\right], \\
V(t)=\bar{p}_{2} e^{-D\left(t-\tau_{1}+n T+\tau_{1}^{*}\right)}, \quad\left(\tau_{1}+n T+\tau_{1}^{*},(n+1) T+\tau_{1}\right] .
\end{array}\right.
$$

So, we obtain $V(t) \leq p$ as $t \rightarrow \infty$. Here $p=\max \left\{\bar{p}_{1}, \bar{p}_{2}\right\}$. According to the definition of $V(t)$, it can be seen that $S_{1}(t)<\frac{p e^{D \tau_{1}}}{\delta_{1}}, S_{2}(t)<\frac{p e^{D \tau_{2}}}{\delta_{2}}, x(t)<p$ as $t \rightarrow \infty$. Let $L=\max \left\{\frac{p e^{D \tau_{1}}}{\delta_{1}}, \frac{p e^{D \tau_{2}}}{\delta_{2}}, p\right\}$. This completes the proof.

\section{Main results}

In this section, we investigate the extinction of the microorganism species, that is, microorganisms are entirely absent from the chemostat permanently, i.e.,

$$
x(t)=0, \quad t \geq 0 .
$$

This is motivated by the fact that $x^{*}=0$ is an equilibrium solution for the variable $x(t)$, as it leaves us with $x^{\prime}(t)=0$. Under these conditions, we show below that the nutrient concentration oscillates with period $T$ in synchronization with the periodic impulsive input of the nutrient concentration.

From the third equation of system (1.1), we have

$$
x^{\prime}(t) \leq e^{-D \tau_{1}} \mu_{1} x\left(t-\tau_{1}\right)+e^{-D \tau_{2}} \mu_{2} x\left(t-\tau_{2}\right)-D x(t) .
$$

By Lemma 2.2, if $e^{-D \tau_{1}} \mu_{1}+e^{-D \tau_{2}} \mu_{2}<D$, then $\lim _{t \rightarrow \infty} x(t)=0$, that is, the microorganism species becomes ultimately extinct. This shows that the specific growth of the microorganism species cannot supply the loss of the microorganism species to flow out no matter 
how much the nutrient input is. Therefore, we assume $e^{-D \tau_{1}} \mu_{1}+e^{-D \tau_{2}} \mu_{2}>D$ in the rest of this paper.

For system (1.1), if we choose $x(t) \equiv 0$ then system (1.1) becomes the following subsystem:

$$
\left\{\begin{array}{l}
\dot{S}_{1}(t)=-D S_{1}(t), \quad t \neq n T, n \in Z_{+}, \\
\dot{S}_{2}(t)=-D S_{2}(t), \quad t \neq n T, n \in Z_{+}, \\
S_{1}\left(t^{+}\right)=S_{1}(t)+p_{1}, \quad t=n T, n \in Z_{+} \\
S_{2}\left(t^{+}\right)=S_{2}(t)+p_{2}, \quad t=n T, n \in Z_{+} .
\end{array}\right.
$$

The subsystem has a unique globally uniform attractive positive $T$-periodic solution $\left(u_{1}^{*}(t), u_{2}^{*}(t)\right)$, which is given in (2.1). Hence, system (1.1) has a $T$-periodic solution $\left(u_{1}^{*}(t), u_{2}^{*}(t), 0\right)$ at which the microorganism culture fails. On the global attractivity of $\left(u_{1}^{*}(t), u_{2}^{*}(t), 0\right)$ for system $(1.1)$, we have the following result.

Theorem 3.1 A periodic solution $\left(u_{1}^{*}(t), u_{2}^{*}(t), 0\right)$ of system (1.1) is globally attractive if

$$
\frac{\mu_{1} e^{-D \tau_{1}} \frac{p_{1}}{1-e^{-D T}}}{k_{1}+\frac{p_{1}}{1-e^{-D T}}}+\frac{\mu_{2} e^{-D \tau_{2}} \frac{p_{2}}{1-e^{-D T}}}{k_{2}+\frac{p_{2}}{1-e^{-D T}}}-D<0 .
$$

Proof Let $\left(S_{1}(t), S_{2}(t), x(t)\right)$ be any solution of system (1.1) with initial values $\left(S_{1}\left(0^{+}\right), S_{2}\left(0^{+}\right)\right.$, $x(0)) \in R_{+}^{3}$, we may choose a sufficiently small positive constant $\varepsilon$ such that

$$
\frac{\mu_{1} e^{-D \tau_{1}} \eta_{1}}{k_{1}+\eta_{1}}+\frac{\mu_{2} e^{-D \tau_{2}} \eta_{2}}{k_{2}+\eta_{2}}<D
$$

where

$$
\eta_{1}=\frac{p_{1}}{1-e^{-D T}}+\varepsilon, \quad \eta_{2}=\frac{p_{2}}{1-e^{-D T}}+\varepsilon .
$$

It follows from that the first and second equation of system (1.1) that $S_{1}^{\prime}(t) \leq S_{1}(t), S_{2}^{\prime}(t) \leq$ $S_{2}(t)$. So we consider the following impulse differential inequalities:

$$
\left\{\begin{array}{l}
\dot{S}_{1}(t) \leq-D S_{1}(t), \quad t \neq n T, n \in Z_{+} \\
\dot{S}_{2}(t) \leq-D S_{2}(t), \quad t \neq n T, n \in Z_{+} \\
S_{1}\left(t^{+}\right)=S_{1}(t)+p_{1}, \quad t=n T, n \in Z_{+} \\
S_{2}\left(t^{+}\right)=S_{2}(t)+p_{2}, \quad t=n T, n \in Z_{+}
\end{array}\right.
$$

By using Lemma 2.1, we have

$$
\begin{aligned}
& \limsup _{t \rightarrow \infty} S_{1}(t) \leq \frac{p_{1}}{1-e^{-D T}}, \\
& \limsup _{t \rightarrow \infty} S_{2}(t) \leq \frac{p_{2}}{1-e^{-D T}} .
\end{aligned}
$$

Hence, there exist a positive integer $n_{1}$ and an arbitrarily small positive constant $\varepsilon$ such that, for all $t \geq n_{1} T$,

$$
\begin{aligned}
& S_{1}(t) \leq \frac{p_{1}}{1-e^{-D T}}+\varepsilon \doteq \eta_{1}, \\
& S_{2}(t) \leq \frac{p_{2}}{1-e^{-D T}}+\varepsilon \doteq \eta_{2} .
\end{aligned}
$$


From (3.5) and the third equation of (1.1) we get, for $t \geq n_{T}+\tau$ where $\tau=\max \left\{\tau_{1}, \tau_{2}\right\}$,

$$
x^{\prime}(t) \leq \frac{\mu_{1} \eta_{1} e^{-D \tau_{1}}}{k_{1}+\eta_{1}} x\left(t-\tau_{1}\right)+\frac{\mu_{2} \eta_{2} e^{-D \tau_{2}}}{k_{2}+\eta_{2}} x\left(t-\tau_{2}\right)-D x(t) .
$$

Consider the following comparison equation:

$$
z^{\prime}(t)=\frac{\mu_{1} \eta_{1} e^{-D \tau_{1}}}{k_{1}+\eta_{1}} z\left(t-\tau_{1}\right)+\frac{\mu_{2} \eta_{2} e^{-D \tau_{2}}}{k_{2}+\eta_{2}} z\left(t-\tau_{2}\right)-D z(t) .
$$

By Lemma 2.3 and (3.3) we obtain

$$
\lim _{t \rightarrow \infty} z(t)=0
$$

Since $x(s)=z(s)>0$ for all $s \in[-\tau, 0]$, by the comparison theorem in differential equations and the nonnegativity of the solution $(x(t) \geq 0)$, we have $x(t) \rightarrow 0$ as $t \rightarrow \infty$.

Without loss of generality, we may assume that $0<x(t)<\varepsilon$, for all $t \geq 0$, by the first equation of system (1.1), we have

$$
S_{1}^{\prime}(t) \geq-\left(D+\frac{\mu_{1} \varepsilon}{\delta_{1} k_{1}}\right) S_{1}(t)
$$

Then we have $\tilde{z}_{1} \leq S_{1}(t)$ and $\tilde{z}_{1} \rightarrow u_{1}^{*}(t)$, as $\varepsilon \rightarrow 0$, where $\tilde{z}_{1}(t)$ is a unique globally asymptotically stable positive periodic solution of

$$
\left\{\begin{array}{l}
z_{1}^{\prime}(t)=-\left(D+\frac{\mu_{1} \varepsilon}{\delta_{1} k_{1}}\right) z_{1}(t), \quad t \neq n T, n \in N \\
z_{1}\left(t^{+}\right)=z_{1}(t)+p_{1}, \quad t=n T, n \in N \\
z_{1}\left(0^{+}\right)=S_{1}\left(0^{+}\right)
\end{array}\right.
$$

From (3.8), we have, for $n T<t<(n+1) T$,

$$
\tilde{z}_{1}(t)=\frac{p_{1} e^{-\left(D+\frac{\mu_{1} \varepsilon}{\delta_{1} k_{1}}\right)(t-n T)}}{1-e^{-\left(D+\frac{\mu_{1} \varepsilon}{\delta_{1} k_{1}}\right) T}} .
$$

By using the comparison theorem of impulsive equations, for any $\varepsilon_{1}>0$ there exists such a $T_{1}>0$ that, for $t>T_{1}$,

$$
S_{1}^{\prime}(t)>\tilde{z}_{1}(t)-\varepsilon_{1}
$$

On the other hand, from the first equation of (1.1), it follows that

$$
S_{1}^{\prime}(t) \leq-D S_{1}(t)
$$

Consider the following comparison system:

$$
\left\{\begin{array}{l}
z_{2}^{\prime}(t)=-D z_{2}(t), \quad t \neq n T, n \in N \\
z_{2}\left(t^{+}\right)=z_{2}(t)+p_{1}, \quad t=n T, n \in N \\
z_{2}\left(0^{+}\right)=S_{1}\left(0^{+}\right)
\end{array}\right.
$$


Then we have

$$
S_{1}(t)<\tilde{z}_{2}+\varepsilon_{1},
$$

as $t \rightarrow \infty$ and $\tilde{z}_{2}(t)=u^{*}(t)$, where $\tilde{z}_{2}(t)$ is a unique positive solution of (3.10).

Let $\varepsilon \rightarrow \infty$, then it follows from (3.9) and (3.11) that

$$
u_{1}^{*}(t)-\varepsilon_{1}<S_{1}(t)<u_{1}^{*}(t)+\varepsilon_{1}
$$

for $t$ large enough, which implies $S_{1}(t) \rightarrow u_{1}^{*}(t)$ as $t \rightarrow \infty$.

By a similar argument to the above, we know $S_{2}(t) \rightarrow u_{2}^{*}(t)$ as $t \rightarrow \infty$. This completes the proof.

Theorem 3.2 The system (1.1) is permanent, if

$$
p_{1}>\frac{D k_{1}\left(e^{D T}-1\right)}{\mu_{1} e^{-D \tau_{1}}-D}
$$

or

$$
p_{2}>\frac{D k_{2}\left(e^{D T}-1\right)}{\mu_{2} e^{-D \tau_{2}}-D} .
$$

Proof Let $\left(S_{1}(t), S_{2}(t), x(t)\right)$ be any positive solution of system (1.1). Without loss of generality, we may assume that

$$
p_{1}>\frac{D k_{1}\left(e^{D T}-1\right)}{\mu_{1} e^{-D \tau_{1}}-D} .
$$

So there is $m_{1}$ such that

$$
0<m_{1}<\frac{\delta_{1} k_{1}}{\mu_{1}}\left(\frac{1}{T} \ln \frac{p\left(\mu_{1} e^{-D \tau_{1}}-D\right)+D k_{1}}{D k_{1}}-D\right) .
$$

From the third equation of system (1.1), we have

$$
x^{\prime}(t) \geq\left(\mu_{1} e^{-D \tau_{1}} \frac{S_{1}(t)}{k_{1}+S_{1}(t)}-D\right) x(t)-\mu_{1} e^{-D \tau_{1}} \frac{d}{d t} \int_{t-\tau_{1}}^{t} \frac{S_{1}(\theta) x(\theta)}{k_{1}+S_{1}(\theta)} d \theta .
$$

Let

$$
V(t)=x(t)+\mu_{1} e^{-D \tau_{1}} \int_{t-\tau_{1}}^{t} \frac{S_{1}(\theta) x(\theta)}{k_{1}+S_{1}(\theta)} d \theta .
$$

Calculating the derivative of $V(t)$ along the solution of (1.1), it follows from (3.15) that

$$
V^{\prime}(t) \geq D\left(\mu_{1} e^{-D \tau_{1}} \frac{S_{1}(t)}{D\left(k_{1}+S_{1}(t)\right)}-1\right) x(t)
$$

For the above $m_{1}$ we can choose a positive constant $\varepsilon_{1}$ small enough such that

$$
\frac{\mu_{1} e^{-D \tau_{1}} \psi_{1}}{D\left(k_{1}+\psi_{1}\right)}>1
$$


where

$$
\psi_{1}=\frac{p_{1} e^{-\left(D+\frac{\mu_{1} m_{1}}{\delta_{1} k_{1}}\right) T}}{1-e^{-\left(D+\frac{\mu_{1} m_{1}}{\delta_{1} k_{1}}\right) T}}-\varepsilon_{1}>0 .
$$

For any positive constant $t_{0}$, we claim that the inequality $x(t)<m_{1}$ cannot hold for all $t \geq 0$. Otherwise, there is a positive constant $t_{0}$, such that $t \geq t_{0}$. From the first and fourth equations of system (1.1), we have

$$
\left\{\begin{array}{l}
\dot{S}_{1}(t) \geq-D S_{1}(t)-\frac{\mu_{1} S_{1}(t) m_{1}}{\delta_{1} k_{1}}, \quad t \neq n T, n \in Z_{+}, \\
S_{1}\left(t^{+}\right)=S_{1}(t)+p_{1}, \quad t=n T, n \in Z_{+} .
\end{array}\right.
$$

By Lemma 2.1 there exists such a $T_{1} \geq t_{0}+\tau_{1}$, for $t \geq T_{1}$, that

$$
S_{1}(t)>\frac{p_{1} e^{-\left(D+\frac{\mu_{1} m_{1}}{\delta_{1} k_{1}}\right) T}}{1-e^{-\left(D+\frac{\mu_{1} m_{1}}{\delta_{1} k_{1}}\right) T}}-\varepsilon_{1} \doteq \psi_{1} .
$$

From (3.18) and (3.16), we have

$$
V^{\prime}(t)>D\left(\mu_{1} e^{-D \tau_{1}} \frac{\psi_{1}}{D\left(k_{1}+\psi_{1}\right)}-1\right) x(t)
$$

For all $t \geq T_{1}$, let

$$
x^{l}=\min _{t \in\left[T_{1}, T_{1}+\tau_{1}\right]} x(t)
$$

We show that $x(t) \geq x^{l}$ for all $t \geq T_{1}$. Otherwise, there exists a nonnegative constant $T_{2}$ such that $x(t) \geq x^{l}$ for $t \in\left[T_{1}, T_{1}+\tau_{1}+T_{2}\right], x\left(T_{1}+\tau_{1}+T_{2}\right)=x^{l}$, and $x^{\prime}\left(T_{1}+\tau_{1}+T_{2}\right) \leq 0$. Thus from the third equation of (1.1) and (3.17), we easily see that

$$
\begin{aligned}
x^{\prime}\left(T_{1}+\tau_{1}+T_{2}\right) & >\left[\mu_{1} e^{-D \tau_{1}} \frac{\psi_{1}}{k_{1}+\psi_{1}}-D\right] x^{l} \\
& =D\left[\mu_{1} e^{-D \tau_{1}} \frac{\psi_{1}}{D\left(k_{1}+\psi_{1}\right)}-1\right] x^{l} \\
& >0
\end{aligned}
$$

which is a contradiction. Hence we get $x(t) \geq x^{l}>0$ for all $t \geq T_{1}$. From (3.19), we have

$$
V^{\prime}(t)>D\left(\mu_{1} e^{-D \tau_{1}} \frac{\psi_{1}}{D\left(k_{1}+\psi_{1}\right)}-1\right) x^{l}>0
$$

which implies $V(t) \rightarrow+\infty$ as $t \rightarrow \infty$. This is a contradiction to $V(t) \leq L\left(1+\mu_{1} \tau_{1} e^{-D \tau_{1}}\right)$. Therefore, for any positive constant $t_{0}$, the inequality $x(t)<m_{1}$ cannot hold for all $t>t_{0}$. On the one hand, if $x(t) \geq m_{1}$ holds true for all $t$ large enough, then our aim is obtained. On the other hand, $x(t)$ is oscillatory about $m_{1}$. Let

$$
m_{2}=\min \left\{\frac{m_{1}}{2}, m_{1} e^{-D \tau_{1}}\right\}
$$


In the following, we shall show that $x(t) \geq m_{2}$. There exist two positive constants $\bar{t}, \omega$ such that

$$
x(\bar{t})=x(\bar{t}+\omega)=m_{1}
$$

and

$$
x(t)<m_{1} \text { for } \bar{t}<t<\bar{t}+\omega .
$$

When $\bar{t}$ is large enough, the inequality $S_{1}(t)>\psi_{1}$ holds true for $\bar{t}<t<\bar{t}+\omega$. Since $x(t)$ is continuous and bounded and is not affect by the impulse, we conclude that $x(t)$ is uniformly continuous. Hence there exists a constant $T_{3}$ (with $0<T_{3}<\tau_{1}$ and $T_{3}$ independent of the choice $\bar{t})$ such that $x(t)>\frac{m_{1}}{2}$ for all $\bar{t}<t<\bar{t}+T_{3}$. If $\omega \leq T_{3}$, our aim is obtained. If $T_{3}<\omega<\tau_{1}$, from the third equation of (1.1) we have $x(t) \geq-D x(t)$ for $\bar{t}<t \leq \bar{t}+\omega$. Then we have $x(t) \geq m_{1} e^{-D \tau_{1}}$ for $\bar{t}<t<\bar{t}+\omega \leq \bar{t}+\tau_{1}$ since $x(t)=m_{1}$. It is clear that $x(t) \geq m_{2}$ for $\bar{t}<t \leq \bar{t}+\omega$. If $\omega>\tau_{1}$, then we have $x(t)>m_{2}$ for $\bar{t}<t \leq \bar{t}+\tau_{1}$. We show that $x(t) \geq m_{2}$ for all $\bar{t}+\tau_{1} \leq t \leq \bar{t}+\omega$. Otherwise, there exists a nonnegative constant $\bar{t}_{2}$ such that $x(t) \geq m_{2}$ for $t \in\left[\bar{t}+\tau_{1}, \bar{t}+\omega\right], x\left(\bar{t}+\tau_{1}+\bar{t}_{2}\right)=m_{2}$ and $x^{\prime}\left(\bar{t}+\tau_{1}+\bar{t}_{2}\right) \leq 0$. Thus from the third equation of (1.1) and (3.17), we easily see that

$$
\begin{aligned}
x^{\prime}\left(\bar{t}+\tau_{1}+\bar{t}_{2}\right) & >\left[\mu_{1} e^{-D \tau_{1}} \frac{\psi_{1}}{k_{1}+\psi_{1}}-D\right] m_{2} \\
& =D\left[\mu_{1} e^{-D \tau_{1}} \frac{\psi_{1}}{D\left(k_{1}+\psi_{1}\right)}-1\right] m_{2} \\
& >0
\end{aligned}
$$

which is a contradiction. Hence we get $x(t) \geq m_{2}>0$ for all $t \in\left[\bar{t}+\tau_{1}, \bar{t}+\omega\right]$.

Since the interval $[\bar{t}, \bar{t}+\omega]$ is arbitrarily chosen (we only need $\bar{t}$ to be large), we get $x(t) \geq$ $m_{2}$ for $t$ large enough. In view of our arguments above, the choice of $m_{2}$ is independent of the positive solution of (1.1) which satisfies $x(t) \geq m_{2}$ for sufficiently large $t$.

By Lemma 2.3, we have $x(t) \leq L$ for $t \geq 0$. Hence, from the first equation of (1.1), we have

$$
S^{\prime}(t) \geq-\left(D+\frac{\mu_{1} L}{\delta_{1} k_{1}}\right) S_{1}(t)
$$

Then we have $S_{1}(t) \geq \tilde{z}_{3}(t)$, where $\tilde{z}_{3}$ is a unique globally asymptotically stable positive periodic solution of

$$
\left\{\begin{array}{l}
z_{3}^{\prime}(t)=-\left(D+\frac{\mu_{1} L}{\delta_{1} k_{1}}\right) z_{3}(t), \quad t \neq n T, n \in N \\
z_{3}\left(t^{+}\right)=z_{3}(t)+p_{1}, \quad t=n T, n \in N \\
z_{3}\left(0^{+}\right)=S_{1}\left(0^{+}\right)
\end{array}\right.
$$

There exists a $\varepsilon>0$ small enough such that, for sufficiently large $t$,

$$
S_{1}(t) \geq \tilde{z}_{3}(t)-\varepsilon \geq \frac{p_{1} e^{-\left(D+\frac{\mu_{1} L}{\delta_{1} k_{1}}\right) T}}{1-e^{-\left(D+\frac{\mu_{1} L}{\delta_{1} k_{1}}\right) T}}-\varepsilon \doteq m_{3} .
$$


By a similar argument, there exists a positive constant $m_{4}$ such that

$$
S_{2}(t) \geq m_{4} .
$$

The proof is complete.

\section{Numerical analysis and discussion}

In this paper, we introduce a growth time delay and pulse input nutrient into the Monod type chemostat model, and theoretically analyze the influence of them on the extinction of the population of the microorganism and the permanence of the system. In Section 3, we give the conditions for extinction and permanence of the microorganisms. Our main results show that if the impulsive periodic nutrient concentration inputs $p_{1}$ and $p_{2}$ are under a certain value, then the population of microorganisms will be eventually extinct. Contrarily, if the impulsive periodic nutrient concentration input $p_{1}$ or $p_{2}$ is over a certain value, it will be permanent. In this case, the microorganism is kept.

In the following, we substantiate the above results by numerical analysis. Then we arbitrarily consider a hypothetical set of parameter values as $\mu_{1}=8, \mu_{2}=10, \delta_{1}=1, \delta_{2}=1$, $D=0.75, \tau_{1}=0.1, \tau_{2}=0.1, T=1, k_{1}=9, k_{2}=4$. If $p_{1}=0.1, p_{2}=0.1$, then Theorem 3.1 holds true, which implies that the microorganism species is extinct (see Figure 1(i)-(iv)). If $p_{1}=2, p_{2}=2$, then Theorem 3.2 holds true, which implies that the microorganism species is permanent (see Figure 2(i)-(iv)).

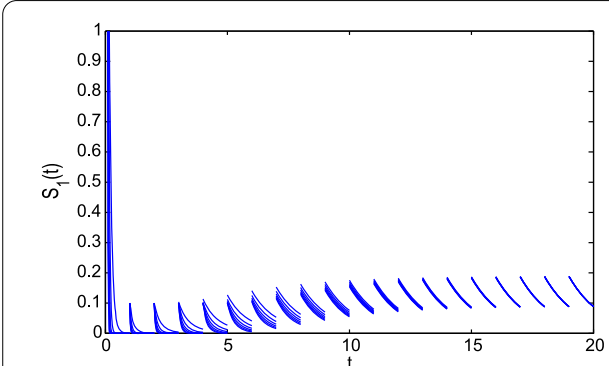

(i)

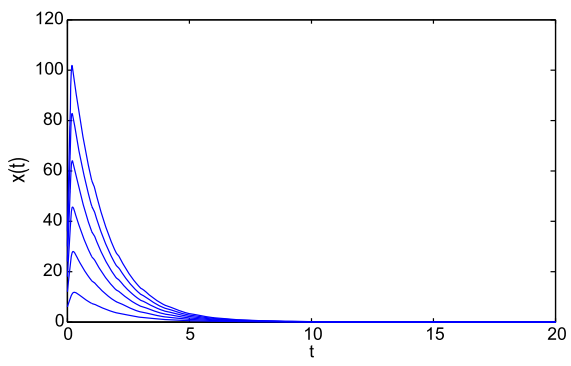

(iii)

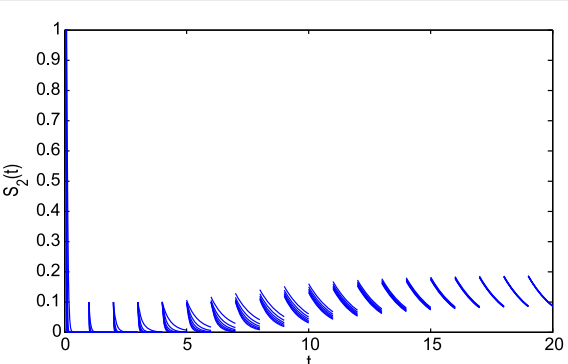

(ii)

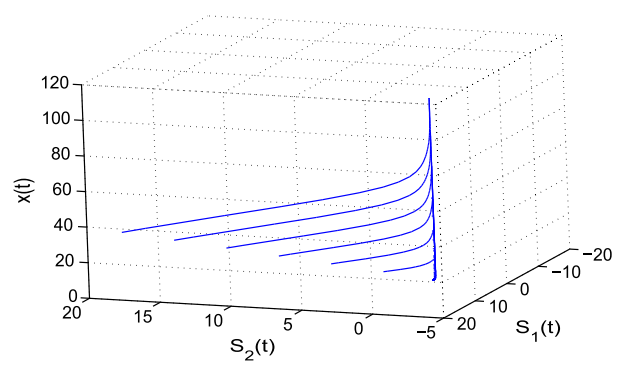

(iv)

Figure 1 Behavior and phase portrait of system (1.1). Dynamical behavior of the system (1.1) with $\mu_{1}=8$, $\mu_{2}=10, \delta_{1}=1, \delta_{2}=1, D=0.75, \tau_{1}=0.1, \tau_{2}=0.1, T=1, p_{1}=0.1, p_{2}=0.1, k_{1}=9, k_{2}=4$. (i) Time-series of the nutrient $S_{1}(t)$ for periodic oscillation. (ii) Time-series of nutrient $S_{2}(t)$ for periodic oscillation. (iii) Time-series of the microorganism population $x(t)$ for extinction. (iv) Phase portrait of $S_{1}(t), S_{2}(t), x(t)$. 


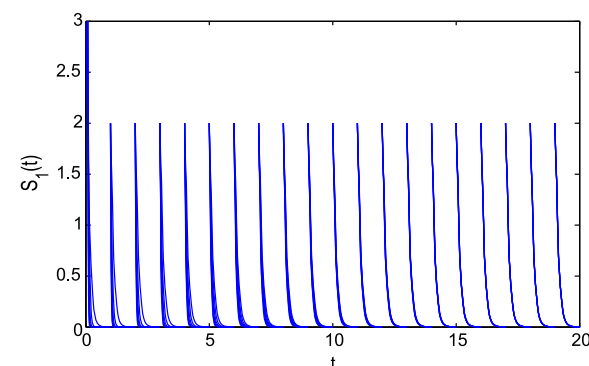

(i)

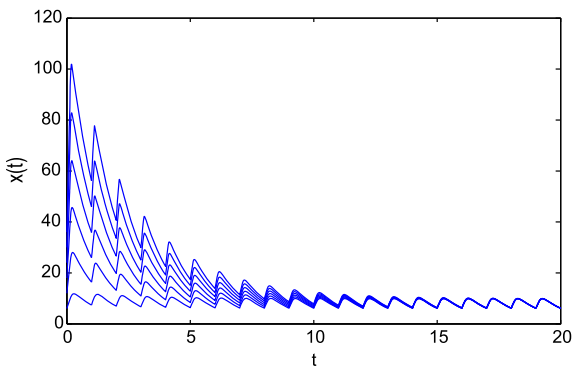

(iii)

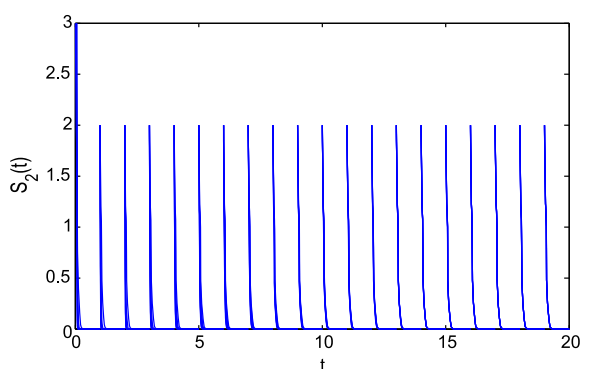

(ii)

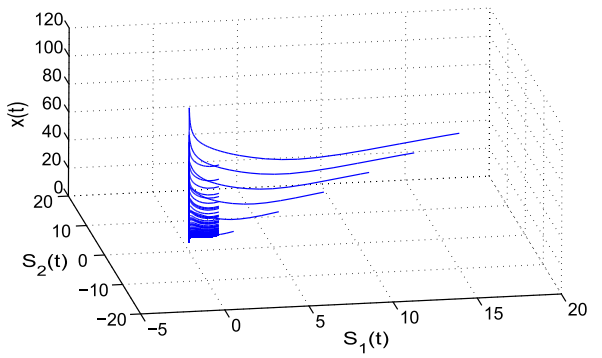

(iv)

Figure 2 Behavior and phase portrait of system (1.1). Dynamical behavior of the system (1.1) with $\mu_{1}=8$, $\mu_{2}=10, \delta_{1}=1, \delta_{2}=1, D=0.75, \tau_{1}=0.1, \tau_{2}=0.1, T=2, p_{1}=2, p_{2}=2, k_{1}=9, k_{2}=4$. (i) Time-series of the nutrient $S_{1}(t)$ for periodic oscillation. (ii) Time-series of nutrient $S_{2}(t)$ for periodic oscillation. (iii) Time-series of the microorganism population $x(t)$ for extinction. (iv) Phase portrait of $S_{1}(t), S_{2}(t), x(t)$.

\section{Competing interests}

The authors declare that they have no competing interests.

\section{Authors' contributions}

All authors completed the paper together. All authors read and approved the final manuscript.

\section{Author details}

${ }^{1}$ College of Mathematics and Information Science, Hebei University, Baoding, 071002, P.R. China. ${ }^{2}$ College of Electronic and Information Engineering, Hebei University, Baoding, 071002, P.R. China.

\section{Acknowledgements}

We would like to thank the reviewers for their valuable comments and suggestions on the manuscript. This work was supported by the National Natural Science Foundation of China (No. 11271106), Natural Science Foundation of Hebei Province of China (No. A2013201232) and the Youth Foundation of Hebei University (No. 2014-295).

\section{Received: 27 March 2015 Accepted: 26 August 2015 Published online: 09 October 2015}

\section{References}

1. Hale, JK, Somolinos, AS: Competition for fluctuating nutrient. J. Math. Biol. 18, 255-280 (1983)

2. Hsu, SB: A competition model for a seasonally fluctuating nutrient. J. Math. Biol. 9, 115-132 (1980)

3. Alessandra, G, Oscar, DF, Sergio, R: Food chains in the chemostat: relationships between mean yield and complex dynamics. Bull. Math. Biol. 60, 703-719 (1998)

4. Mark, K, Sayler, GS, Waltman, TW: Complex dynamics in a model microbial system. Bull. Math. Biol. 54, 619-648 (1992)

5. Eri, ZF, Mark, K: Invasion and chaos in a pulsed mass-action chemostat. Theor. Popul. Biol. 44, 203-224 (1993)

6. Jiao, JJ, Cai, SH: A delayed chemostat model with impulsive diffusion and input on nutrients. Adv. Differ. Equ. 2009, Article ID 514240 (2009). doi:10.1155/2009/514240

7. Jiao, JJ, Ye, KL, Chen, LS: Dynamical analysis of a five-dimensioned chemostat model with impulsive diffusion and pulse input environmental toxiant. Chaos Solitons Fractals 44, 17-27 (2011)

8. Butler, GJ, Hsu, SB, Waltman, P: A mathematical model of the chemostat with periodic washout rate. SIAM J. Appl. Math. 45, 435-449 (1985)

9. Lenas, P, Pavlou, S: Coexistence of three competing microbial populations in a chemostat with periodically varying dilution rate. Math. Biosci. 129, 111-142 (1995)

10. DeAnglis, DL, Goldstein, RA, O'Neill, RV: A model for trophic interaction. Ecology 56, 881-892 (1975)

11. Jang, S: Dynamics of variable-yield nutrient-phytoplankton-zooplankton models with nutrient recycling and self-shading. J. Math. Biol. 40, 229-250 (2000) 
12. Fu, G, Ma, W: Hopf bifurcations of a variable yield chemostat model with inhibitory exponential substrate uptake. Chaos Solitons Fractals 30, 845-850 (2006)

13. Ruan, SG, Wolkowicz, GSK: Bifurcation analysis of a chemostat model with a distributed delay. J. Math. Anal. Appl. 204, 786-812 (1996)

14. Thingstad, TF, Langeland, TI: Dynamics of chemostat culture: the effect of a delay in cell response. J. Theor. Biol. 48, 149-159 (1974)

15. Ellermeyer, SF: Competition in the chemostat: global asymptotic behavior of a model with delayed response in growth. SIAM J. Appl. Math. 54, 456-465 (1994)

16. Wolkowicz, GSK, Xia, H: Global asymptotic behavior of a chemostat model with discrete delays. SIAM J. Appl. Math. 57, 1019-1043 (1997)

17. Wolkowicz, GSK, Xia, H, Ruan, SG: Competition in the chemostat: a distributed delay model and its global asymptotic behavior. SIAM J. Appl. Math. 57, 1281-1310 (1997)

18. Xia, H, Wolkowicz, GSK, Wang, L: Transient oscillations induced by delayed growth response in the chemostat. J. Math. Biol. 50, 489-530 (2005)

19. Zhao, T: Global periodic solutions for a differential delay system modelling a microbial population in the chemostat. J. Math. Anal. Appl. 193, 329-352 (1995)

20. Meng, X, Li, Z, Nieto, JJ: Dynamic analysis of Michaelis-Menten chemostat-type competition models with time delay and pulse in a polluted environment. J. Math. Chem. 47, 123-144 (2009)

21. Jiao, JJ, Yang, $X$, Chen, LS, Cai, S: Effect of delayed response in growth on the dynamics of a chemostat model with impulsive input. Chaos Solitons Fractals 42, 2280-2287 (2009)

22. Jiao, JJ, Chen, LS: Dynamical analysis of a chemostat model with delayed response in growth and pulse input in polluted environment. J. Math. Chem. 46, 502-513 (2009)

23. Caperon, J: Time lag in population growth response of Isochrysis galbana to a variable nitrate environment. Ecology 50, 188-192 (1969)

24. Bush, AW, Cook, AE: The effect of time delay and growth rate inhibition in the bacterial decomposition of waste water. J. Theor. Biol. 63, 385-395 (1975)

25. Bainov, DD Simeonov, PS: Impulsive Differential Equations: Periodic Solutions and Applications. Longman, Harlow (1993)

26. Lakshmikantham, V Bainov, DD, Simeonov, PS: Theory of Impulsive Differential Equations. World Scientific, Singapore (1989)

27. Kuang, Y: Delay Differential Equations with Applications in Population Dynamics. Academic Press, San Diego (1993)

\section{Submit your manuscript to a SpringerOpen ${ }^{\circ}$ journal and benefit from:}

- Convenient online submission

- Rigorous peer review

- Immediate publication on acceptance

- Open access: articles freely available online

- High visibility within the field

- Retaining the copyright to your article 